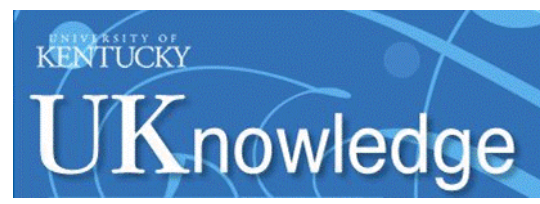

University of Kentucky

UKnowledge

$11-7-2017$

\title{
Universality of Local Dissipation Scales in Turbulent Boundary Layer Flows With and Without Free-Stream Turbulence
}

\author{
Sabah F. H. Alhamdi \\ University of Kentucky, sabahalbatat@uky.edu \\ Sean C. C. Bailey \\ University of Kentucky, sean.bailey@uky.edu
}

Follow this and additional works at: https://uknowledge.uky.edu/me_facpub

Part of the Fluid Dynamics Commons, and the Mechanical Engineering Commons

Right click to open a feedback form in a new tab to let us know how this document benefits you.

\section{Repository Citation}

Alhamdi, Sabah F. H. and Bailey, Sean C. C., "Universality of Local Dissipation Scales in Turbulent Boundary Layer Flows With and Without Free-Stream Turbulence" (2017). Mechanical Engineering Faculty Publications. 38.

https://uknowledge.uky.edu/me_facpub/38

This Article is brought to you for free and open access by the Mechanical Engineering at UKnowledge. It has been accepted for inclusion in Mechanical Engineering Faculty Publications by an authorized administrator of UKnowledge. For more information, please contact UKnowledge@lsv.uky.edu. 


\section{Universality of Local Dissipation Scales in Turbulent Boundary Layer Flows With}

and Without Free-Stream Turbulence

Digital Object Identifier (DOI)

https://doi.org/10.1063/1.4996200

\section{Notes/Citation Information}

Published in Physics of Fluids, v. 29, issue 11, 115103, p. 1-10.

This article may be downloaded for personal use only. Any other use requires prior permission of the author and AIP Publishing.

The following article appeared in Physics of Fluids, v. 29, issue 11, 115103, p. 1-10 and may be found at https://doi.org/10.1063/1.4996200. 


\title{
Universality of local dissipation scales in turbulent boundary layer flows with and without free-stream turbulence
}

\author{
Sabah F. H. Alhamdi ${ }^{1,2}$ and Sean C. C. Bailey ${ }^{1, a)}$ \\ ${ }^{1}$ Department of Mechanical Engineering, University of Kentucky, Lexington, Kentucky 40506, USA \\ ${ }^{2}$ University of Misan, Amarah, Misan, Iraq
}

(Received 14 July 2017; accepted 21 October 2017; published online 7 November 2017)

\begin{abstract}
Measurements of the small-scale dissipation statistics of turbulent boundary layer flows with and without free-stream turbulence are reported for $R e_{\tau} \approx 1000\left(\operatorname{Re}_{\theta} \approx 2000\right)$. The scaling of the dissipation scale distribution is examined in these two boundary conditions. Results demonstrated that the local large-scale Reynolds number based on the measured longitudinal integral length scale fails to properly normalize the dissipation scale distribution near the wall in these two free-stream conditions due to the imperfect characterization of the upper bound of the inertial cascade by the integral length scale. A surrogate found from turbulent kinetic energy and mean dissipation rate only moderately improved the scaling of the dissipation scales, relative to the measured integral length scale. When a length scale based on the distance from the wall [as suggested by Bailey and Witte, "On the universality of local dissipation scales in turbulent channel flow," J. Fluid Mech. 786, 234-252 (2015)] was utilized to scale the dissipation scale distribution, in the region near the wall, there was a noticeable improvement in the collapse of the normalized distribution of dissipation scales. In addition, unlike in channel flows, in the outer layer of the turbulent boundary layer, the normalized distributions of the local dissipation scales were observed to be dependent on the wall-normal position. This was found to be attributable to the presence of external intermittency in the outer layer as the presence of free-stream turbulence was found to restore the scaling behavior by replacing the intermittent laminar flow with turbulent flow. Published by AIP Publishing. https://doi.org/10.1063/1.4996200
\end{abstract}

\section{INTRODUCTION}

One of the most significant theories in the study of turbulence is the universal equilibrium hypothesis of Kolmogorov, ${ }^{14}$ which postulates that the small scales of turbulence are homogeneous and statistically isotropic and that, due to a cascade of kinetic energy from the largest scales of turbulence to the smallest scales, the smallest scales become disconnected from the boundary conditions and thus become uniquely and universally dependent only on the mean rate of dissipation of turbulent kinetic energy, $\langle\varepsilon\rangle$, and the kinematic viscosity, $v$. Note that here \langle\rangle denotes an ensemble-averaged quantity and $\varepsilon$ can be estimated through

$$
\varepsilon=\frac{v}{2}\left(\frac{\partial u_{i}}{\partial x_{j}}+\frac{\partial u_{j}}{\partial x_{i}}\right)^{2}
$$

in which $u_{i}$ refers to the fluctuating components of the velocity vector, given by $u_{i}\left(x_{j}, t\right)=U_{i}\left(x_{j}, t\right)-\left\langle U_{i}\left(x_{j}, t\right)\right\rangle$, where $t$ denotes time, $x_{j}$ indicates spatial location, and $U_{i}$ denotes the components of the instantaneous local velocity vector. Through dimensional analysis of $\langle\varepsilon\rangle$ and $v$, length, velocity, and time scales corresponding to the dissipation of kinetic energy can be formed: the Kolmogorov dissipation length scale, $\eta_{K}$ $\sim\left(v^{3} /\langle\varepsilon\rangle\right)^{1 / 4}$; the Kolmogorov velocity scale, $u_{K}=(v\langle\varepsilon\rangle)^{1 / 4}$; and the Kolmogorov time scale, $\tau_{K}=(v /\langle\varepsilon\rangle)^{1 / 2}$. The existence of a universal equilibrium region was heavily tested in the succeeding decades, and, as a result, there is a great

a)Electronic mail: sean.bailey@uky.edu amount of evidence to support Kolmogorov's concept of smallscale universality, most notably through the collapse of the energy spectra scaled by $v,\langle\varepsilon\rangle$, and $\eta_{K}$ in the dissipation region, e.g., Grant et al., ${ }^{10}$ Saddoughi and Veeravalli, ${ }^{22}$ and others.

Key to these theories is the energy cascade process by which energy is transferred from large energy-producing eddies, described by the integral length scale, $L$, down to the smallest eddies, characterized by $\eta_{K}$. Given the sufficient separation of these scales, within the universal equilibrium range, there will be an inertial subrange where the turbulent dynamics depend only on $\langle\varepsilon\rangle$ and not on $v$. When the spatial separation between two points in space, represented by a vector with components $r_{j}$, lies in this inertial subrange such that $L \gg|r| \gg \eta_{K}$, the longitudinal structure function of the streamwise velocity, $S_{n}$, should follow power-law behavior such that

$$
S_{n} \equiv\left\langle\left(\delta_{r} u\right)^{n}\right\rangle=A_{n}\left(\frac{|r|}{L}\right)^{\zeta_{n}},
$$

where $A_{n}$ are universal constants and $\delta_{r} u$ represents the longitudinal velocity increment defined as

$$
\delta_{r} u \equiv\left(u_{i}\left(x_{j}+r_{j}\right)-u_{i}\left(x_{j}\right)\right)\left(\frac{r_{i}}{|r|}\right) .
$$

Kolmogorov's theory indicated that $\zeta_{n}=n / 3$. However, experimental investigations, e.g., the work of Anselmet et al., ${ }^{2}$ have shown that $\zeta_{n}$ differs from this linear scaling and has nonlinear dependence on $n$. This deviation from the expected behavior has long been attributed to spatial intermittency in the fine 
structure of the turbulent flow, as reviewed by Frisch, ${ }^{9}$ for example. In other words, the dissipation does not occur homogeneously in space but instead occurs in compact regions in space, separated by regions of little-to-no dissipation.

This intermittency persists throughout the universal equilibrium range, and, as a result, the use of a singular mean dissipation length scale to describe the turbulent dynamics does not appear to be sufficient. ${ }^{7}$ In this context, an alternative description of the dissipation scale that incorporates the existence of an entire continuum of local dissipation scales becomes attractive. Yakhot ${ }^{30}$ proposed an approach that connects and defines a local scale $\eta$ using the velocity increment across that scale, $\delta_{\eta} u$, whereby

$$
\eta\left|\delta_{\eta} u\right| \sim v,
$$

and $\delta_{\eta} u$ is calculated from Eq. (3) with $|r|=\eta$. This is analogous to the definition of a local Reynolds number based on the local scale $\eta$ and the velocity increment $\delta_{\eta} u$, defining a dissipative scale as the one for which this Reynolds number is $\mathcal{O}(1){ }^{18}$ Yakhot $^{30}$ suggested that this Reynolds number is connected to the crossover scales between the inertial subrange and the viscous dissipation range.

To evaluate these concepts, as $\eta$ is a random field, there is particular interest in characterizing this field through its probability density function (PDF). To address this, Yakhot ${ }^{30}$ presented an analytical description of the PDF of $\eta$. When normalized by $\eta_{0}$, this expression provided good agreement with the PDFs estimated from the high-resolution direct numerical simulation (DNS) data of three-dimensional homogenous isotropic box turbulence of Schumacher. ${ }^{24}$ The scale $\eta_{0}$ is analogous to $\eta_{K}$ and is estimated from $\eta_{0} \approx L R e_{L}^{-0.73}$. $R e_{L}=\left\langle\left|\delta_{L} u\right|\right\rangle L / v$ is a local large-scale Reynolds number which describes the most energetic eddies, with $\delta_{L} u$ determined from Eq. (3) with $|r|=L$. This can be compared to the results of scaling arguments which suggest that $\eta_{K} \approx L R e_{L}^{-0.75}$, and thus the ratio $\eta_{0} / \eta_{K}$ is close to unity, incrementing only gradually as $R e_{L}^{0.02}$ as detailed in the work of Hamlington et al. ${ }^{12}$

The analytical PDF of $\eta / \eta_{0}$, determined by Yakhot, ${ }^{30}$ was compared by Bailey et al. ${ }^{5}$ to PDFs measured in low-Reynoldsnumber turbulent pipe flows at the pipe centerline and within the upper logarithmic layer and those calculated from homogeneous and isotropic DNSs of Schumacher. ${ }^{24}$ The comparison found good qualitative agreement between the experimental results and the analytical description and resulted in the collapse of the measured and simulated PDFs, fortifying the hypothesis that there is universality of the form of the PDFs and hence the distribution of $\eta$.

However, PDFs of $\eta$ were also determined experimentally by Zhou and Xia, ${ }^{32}$ this time in buoyancy-driven turbulence. Instead of finding good agreement between PDFs computed at different positions within the flow and at different Rayleigh numbers, the results exhibited a higher probability of there being scales smaller than $\eta_{0}$ than found by Schumacher ${ }^{24}$ and Bailey et al. ${ }^{5}$ Zhou et al. attributed this discrepancy to a much higher level of small-scale intermittency caused by the presence of thermal plumes, which have a characteristic dimension in a thermal boundary layer that is smaller than $\eta_{K}$.
Hamlington et al. ${ }^{12}$ also computed the PDFs of $\eta / \eta_{0}$ from very high-resolution DNS of turbulent channel flows and determined that universality of the PDF exists for much of the channel, except in the near-wall region. A similar position dependence of the PDF was identified experimentally in freeshear flows by Morshed et al. ${ }^{16}$ who showed that this location dependency is related to large-scale shear through a mean shear-dissipation Reynolds number. In both studies, the comparison of PDFs calculated within regions of reduced shear to those observed in homogenous and nearly homogeneous turbulence by Schumacher ${ }^{24}$ and Bailey et al. ${ }^{5}$ showed good agreement. Hence the presence of mean velocity shear appears to negatively influence the scaling of the PDFs.

Recently, Bailey and Witte ${ }^{4}$ experimentally determined the PDFs of $\eta$ in a turbulent channel flow. They found that when $\eta_{0}$ is used as a normalization parameter, the distributions of PDFs are in good agreement with those previously reported experimentally, numerically, and analytically. However, using $\eta_{0}$ as a scaling parameter in the near-wall region leads to a non-universality of small scales in this region, coinciding with the presence of increased mean shear. Bailey and Witte found that the lack of universality could be attributed to the imperfect description of large scales, $L$, when using the measured integral length scale. This influences the scaling parameter $\eta_{0}$ and results in the small scales being poorly described by $\eta_{0}$. Thus, they defined an alternate scaling parameter, $\eta^{*}$, which depends on a mixing length scale and its corresponding Reynolds number, with the mixing length scale related to the distance from the wall. Using $\eta^{*}$ instead of $\eta_{0}$, Bailey and Witte found there to be an improved collapse of the PDFs near the wall. However, this collapse degraded for $y / \delta>0.5$, which suggested there exists scaling behavior analogous to the innerand outer-scaling that describes the mean flow.

In summary, these recent results imply that the mean shear impacts the description of the local dissipation scales. However, this impact appears to be through the scaling parameter chosen, rather than through the distribution of the PDF itself. In regions of small mean shear such as in homogenous isotropic turbulence, in the centerline of channel and pipe flows, and the center of the Rayleigh-Bernard convection cells, the appropriate scaling parameter appears to be $\eta_{0}$, which is analogous to the Kolmogorov scale. In the high-shear regions of turbulent channel flows, this scale appears to be proportional to the distance from the wall.

The objective of the present research is to investigate further the scaling of the PDFs within wall-bounded flows suggested by Bailey and Witte. ${ }^{4}$ To do this, experiments were conducted in a turbulent boundary layer developing within both laminar and turbulent free streams. These results were used to calculate the PDFs of $\eta$ at various distances from the wall and investigate their scaling behavior.

\section{EXPERIMENT DESCRIPTION}

The experiments were performed in an open circuit wind tunnel flow facility located in the Experimental Fluid Dynamics Laboratory at the University of Kentucky. This facility has a test section with a $0.61 \mathrm{~m} \times 0.61 \mathrm{~m}$ cross-sectional area and a length of $1.2 \mathrm{~m}$ and can achieve free-stream velocities 
up to $45.7 \mathrm{~m} / \mathrm{s}$. For these experiments, the free-stream velocity was $U_{\infty} \approx 4 \mathrm{~m} / \mathrm{s}$. To generate a turbulent boundary layer, a smooth flat plate with dimensions of $886 \mathrm{~mm} \times 608 \mathrm{~mm}$ was placed in the test section. To trip the boundary layer forming on the plate, it was equipped at the leading edge by $50.8 \mathrm{~mm}$ of a 60 grit sandpaper trip. A trailing edge flap was also located on the plate to prevent leading edge flow separation. To produce the free-stream turbulence, a grid with a solidity of 0.32 and square perforations having mesh sizes of $M=25.4 \mathrm{~mm}$ could be inserted in the inlet of the test section. The resulting free-stream turbulence intensity at the measurement location was approximately $2.5 \%$.

To measure the properties of the boundary layer developing along the smooth plate when mounted in the wind tunnel, measurements of streamwise velocity, $U_{1}$, were performed over a range of wall-normal distances, using a hot-wire probe made from a platinum-core Wollaston wire etched to a sensing length of $\ell=0.50 \mathrm{~mm}$ and diameter of $2.5 \mu \mathrm{m}$. This leads to $\ell^{+}=\ell u_{\tau} / v \approx 6$, with $u_{\tau}$ being the friction velocity. The maximum of the ratio $\ell / \eta_{K}$ was $\approx 3$ and occurred in the measurement locations closest to the wall. The probe was operated in a constant temperature anemometer (IFA 300 CTA) system at an overheat ratio of 1.6. Frequency response of the probe was measured via square wave test to be $75 \mathrm{kHz}$. The CTA signal was low-pass filtered at half the sample frequency, $f_{s}$, which was $100 \mathrm{kHz}$ for the case without free-stream turbulence and $200 \mathrm{kHz}$ for the case with free-stream turbulence.

The probe was located $760 \mathrm{~mm}$ from the leading edge of the smooth plate and traversed in the wall-normal direction, i.e., in the $y$-direction, from its initial position approximately $100 \pm 5 \mu \mathrm{m}$ from the wall to its final position $120 \mathrm{~mm}$ from the wall. Streamwise velocity was measured at 40 points logarithmically spaced between these two locations. At each measurement location for the baseline case, the data were sampled for $60 \mathrm{~s}$. For the case with free-stream turbulence, the sample time was increased to $120 \mathrm{~s}$. The free-stream temperature was measured by a type $\mathrm{K}$ thermocouple and found to remain approximately constant for each measurement, changing by less than $0.4{ }^{\circ} \mathrm{C}$ over the course of a profile measurement.

To traverse the probe normal to the plate surface in the wind tunnel, a nano-stepping traverse equipped with a highaccuracy linear encoder and controlled by a stepper motor was used $(0.5 \mu \mathrm{m}$ resolution and $\pm 3 \mu \mathrm{m}$ accuracy). An electrical contact switch was used to set the initial position of the hotwire probe from the wall. At the initial measurement location, the distance from the wall to the probe was found using a distance measuring microscope (Titan Tool Supply 2DM-1 with $\pm 15 \mu \mathrm{m}$ accuracy). Probe positioning and data acquisition were controlled by a custom LabVIEW program.

Hot-wire probe calibrations were performed in the freestream directly prior to, and following, each measurement run using a pitot-static tube located in the free stream at the measurement location. The pre- and post-measurement calibrations were used to verify that there was no voltage drift during a profile measurement. To maximize the sensitivity over the range of calibration velocities, two transducers with an accuracy of $0.25 \%$, having sensitivities of 125 and $1245 \mathrm{~Pa}$, were used to measure the pressure difference between total pressure and static pressure. The calibration data were fitted
TABLE I. Experimental conditions and symbols used to represent each case in the following figures.

\begin{tabular}{lccccccc}
\hline \hline $\begin{array}{l}\text { Free } \\
\text { stream }\end{array}$ & $\operatorname{Re}_{\tau}$ & $\operatorname{Re}_{\theta}$ & $u_{\tau}(\mathrm{m} / \mathrm{s})$ & $\begin{array}{l}v / u_{\tau} \\
(\mu \mathrm{m})\end{array}$ & $\theta(\mathrm{mm})$ & $\delta(\mathrm{mm})$ & Symbol \\
\hline Laminar & 1000 & 1800 & 0.19 & 79 & 6.2 & 82 & $\Delta$ \\
Turbulent & 1000 & 2100 & 0.18 & 83 & 7.1 & 85 & $\square$ \\
\hline \hline
\end{tabular}

with a fourth-order polynomial to convert the measured timedependent voltage into time series of streamwise velocity, $U_{1}(t)$. As the calibrations were being conducted using the lower portion of the pressure transducer ranges, before accepting data from a measurement, the calibration curves from each transducer were verified to be in agreement.

The turbulent boundary layer at the measurement location had Reynolds number, $R e_{\tau}=\delta u_{\tau} / v \approx 1000\left(R e_{\theta}=\theta U_{\infty} / v\right.$ $\approx 2000$ ). Here, $\theta$ is the momentum thickness, and $\delta$ is the boundary layer thickness calculated at the streamwise mean velocity, $\left\langle U_{1}\right\rangle=0.99 U_{\infty}$. Here, $\delta$ is the boundary layer thickness calculated at the streamwise mean velocity, $\left\langle U_{1}\right\rangle$ $=0.99 U_{\infty}$, and $\theta$ is the momentum thickness. The friction velocity, $u_{\tau}$, was calculated by finding the value of $u_{\tau}$ which best scaled the measured velocity profiles in the near-wall region to the DNS data of Schlatter and Örlü. ${ }^{23}$ The experimental conditions for each case are presented in Table I. Note that zero-pressure-gradient conditions were not enforced or verified, and thus it is unlikely that the boundary layer is a true canonical zero-pressure-gradient turbulent boundary layer. However, we do not believe that any deviations from canonical flow conditions should impact the scaling arguments discussed here.

\section{OVERVIEW OF MEASURED SCALES OF TURBULENCE}

To describe the turbulence, we first present examples of the estimated energy and dissipation spectra as well as the measured statistics. The wavenumber spectra $E_{11}\left(k_{1}\right)$ was estimated through

$$
E_{11}\left(k_{1}\right)=\frac{\left\langle U_{1}\right\rangle}{2 \pi} F_{11}\left(\frac{2 \pi}{\left\langle U_{1}\right\rangle} f\right),
$$

in which $F_{11}(f)$ is the frequency, $f$, spectrum calculated from the magnitude of the Fourier transform of the velocity fluctuations $u_{1}=U_{1}(t)-\left\langle U_{1}\right\rangle$. In order to interpret temporal information into spatial information in the calculation, Taylor's frozen flow hypothesis ${ }^{27}$ was used, where the streamwise wavenumber, $k_{1}$, was found from frequency through $2 \pi f_{1} /\left\langle U_{1}\right\rangle$. There is much literature on the validity of Taylor's hypothesis, ${ }^{3,8,15}$ which suggests that possible additional corrections are required when translating the temporal domain into the spatial domain. Such corrections are not attempted here since the focus of the study is the smallest turbulent scales, where Taylor's hypothesis provides a reasonable approximation of the spatial separation. Note also that these corrections are not without problems, especially for the low Reynolds numbers of the present study, where they could lead to bias of the data prior to the analysis. ${ }^{20,21,25}$ 
In order to estimate $\eta_{K}$, an estimate of $\langle\varepsilon\rangle$ must first be found. One approach to calculate $\langle\varepsilon\rangle$ is from the integration of the approximated one-dimensional dissipation spectrum $D\left(k_{1}\right)$ after assuming local isotropy ${ }^{29}$ following

$$
\langle\varepsilon\rangle \approx 15 v \int_{0}^{k_{c}} D\left(k_{1}\right) d k_{1} \approx 15 v \int_{0}^{k_{c}} k_{1}^{2} E_{11}\left(k_{1}\right) d k_{1},
$$

where $D\left(k_{1}\right)$ was approximated from the longitudinal energy spectrum through $D\left(k_{1}\right)=15 v k_{1}^{2} E_{11}\left(k_{1}\right)$. To minimize the effect of the $f^{2}$ noise of the thermal anemometer and prevent contamination of the $\langle\varepsilon\rangle$ estimate by the oversampling of the velocity signals in the present measurements, an appropriate cutoff wavenumber, $k_{c}$, was applied as an upper bound of the integration. This cutoff was set at the wavenumber when an inflection started to appear in the frequency spectrum, i.e., the frequency at which the noise started to overcome the useful signal.

In this work, the streamwise component of the velocity was resolved utilizing a single-sensor thermal anemometry probe, which was not capable of conducting measurements of all the components of the time series of the local rateof-deformation tensor. Hence, an alternate estimate of the dissipation rate could be obtained using the one-dimensional approximation $^{26}$

$$
\varepsilon(t) \approx 15 v\left(\frac{\partial u_{1}}{\partial x_{1}}\right)^{2},
$$

which assumes local homogeneity. However, as reported in the study of Pope, ${ }^{19}$ for example, such alternatives are only estimated to be qualitatively similar to the instantaneous dissipation. To evaluate Eq. (7), Taylor's hypothesis and a first-order finite difference were used as follows:

$$
\varepsilon(t) \approx 15 v \frac{1}{\left\langle U_{1}\right\rangle^{2}}\left[\frac{u_{1}(t+\Delta t)-u_{1}(t)}{\Delta t}\right]^{2},
$$

where $\Delta t=1 / f_{s}$. In the present measurements, to minimize contamination from instrumentation noise, the data were filtered using an additional zero-phase, eight order digital Butterworth filter. The cutoff frequency was chosen to be $k_{c}\left\langle U_{1}\right\rangle / 2 \pi$. Both Eq. (6) and the mean of Eq. (8) were used to estimate $\langle\varepsilon\rangle$, and both were determined to be in agreement. For the remainder of this work, the values calculated using Eq. (8) are the ones presented. Note that the assumptions of local homogeneity and isotropy used to extract surrogates for the three-dimensional dissipation from one-dimensional measurements break down near the wall as evidenced by a breakdown of Kolmogorov scaling at high wavenumbers when using the estimate of $\langle\varepsilon\rangle$ used here. Hence, only measurement points for which $y^{+}=y u_{\tau} / v>25$, where such scaling is observed, are included in the present study.

The measured longitudinal one-dimensional energy spectra and the corresponding approximated one-dimensional dissipation spectra for both the laminar and turbulent free-stream cases are presented in Figs. 1(a) and 1(b). Two different $y$ positions are presented, $y^{+} \approx 30$ and 800 , as they represent the points closest to the wall and at the edge of the outer layer of the boundary layer where the flow is subjected to an interface between the boundary layer and free-stream conditions and therefore intermittently displays the properties of each. The energy and dissipation spectra have been normalized by $\left(\langle\varepsilon\rangle v^{5}\right)^{1 / 4}$ and $\left(\langle\varepsilon\rangle \eta_{K}^{5}\right)$, respectively, and thus scaled using Kolmogorov scaling. As expected, for the cases where the flow is fully turbulent (near the wall and at the edge of the boundary layer for the case with a turbulent free stream, and thus, the external intermittency is between boundary layer and free-stream turbulence), the scaled energy spectra follow Kolmogorov scaling at high wavenumbers. For the measurement in the outer region of the boundary layer when the free stream is laminar and the external intermittency is between boundary layer turbulence and laminar flow, this scaling does not hold and the corresponding spectra does not monotonically decay and deviate from Kolmogorov scaling at high wavenumbers. Note that as can be expected for the relatively low Reynolds numbers investigated here, there is no evidence of an inertial subrange.

The corresponding estimated one-dimensional dissipation spectra, shown in Fig. 1(b), provide confidence that the entire dissipation range has been captured by the measurements. Whereas for the case where the flow is consistently turbulent, the dissipation spectra appear log-normal, when laminarturbulent external intermittency is present, the approximated one-dimensional dissipation spectra has a different appearance, being skewed toward larger scales and showing more content at wavenumbers above $\eta_{K}$.

Comparison of the wall-normal dependence of the turbulent statistics measured for the laminar and turbulent free
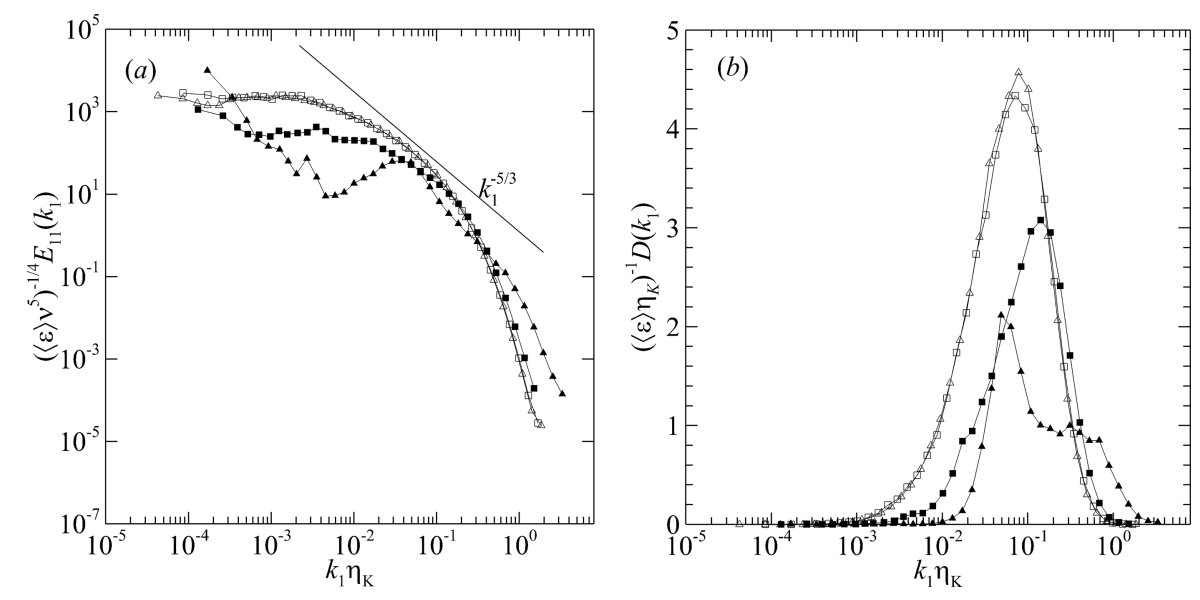

FIG. 1. (a) Normalized longitudinal one-dimensional energy spectra measured at $y^{+} \approx 30$ (hollow symbols) and 800 (filled symbols). (b) Corresponding estimate of the dissipation spectra. Symbols are as in Table I. 

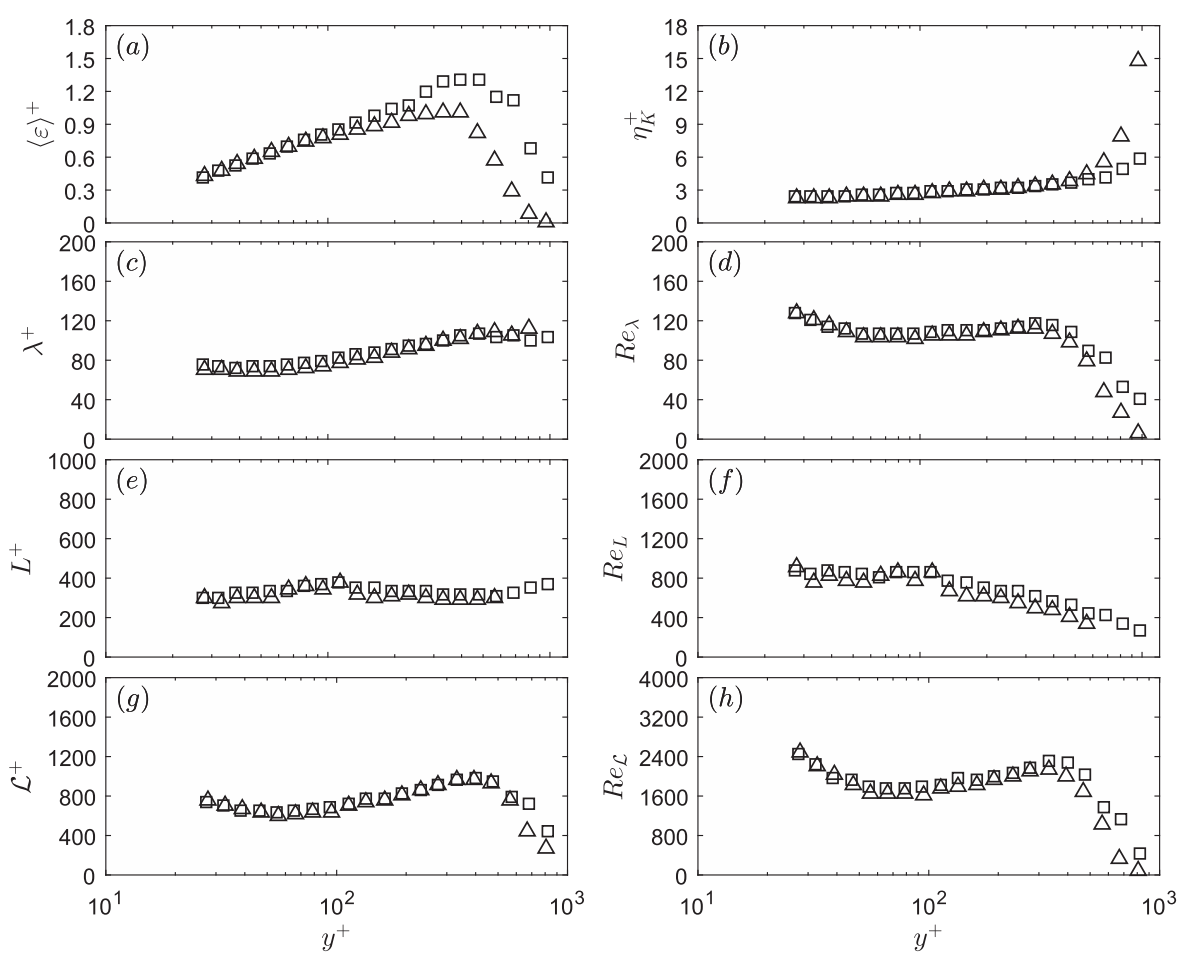

FIG. 2. Wall-normal dependence of (a) the mean dissipation rate; (b) the Kolmogorov scale; (c) the Taylor microscale; (d) the Taylor Reynolds number; (e) the integral length scale; (f) the large-scale Reynolds number; (g) the alternative integral length scale; and (h) the alternative large-scale Reynolds number. Symbols are as in Table I. stream boundary layers is presented in Fig. 2 with the innerscaled dissipation, $\langle\varepsilon\rangle^{+}=\langle\varepsilon\rangle 0.4 / u_{\tau}^{3}$, and the Kolmogorov scale, $\eta_{K}^{+}=\eta_{K} u_{\tau} / v$, presented in Figs. 2(a) and 2(b), respectively. In Fig. 2(a), the inner-scaled profiles of dissipation for both cases increase with wall-normal distance at the same rate up to $y^{+} \approx 350$. At locations further from the wall, the mean dissipation rate of the case without free-stream turbulence decreases rapidly with increasing distance from the wall until reaching zero at the edge of the boundary layer to match the dissipation rate of the laminar free stream. Conversely, for the boundary layer in the turbulent free-stream, there is always turbulence present, so the dissipation rate maximizes at $y^{+} \approx 500$, above which the dissipation rate decreases down to the free-stream levels. Comparison of the mean dissipation rate indicates that the differences in the free-stream conditions largely influence the fine scale behavior only in the outer layer. The values of $\eta_{K}^{+}=\eta_{K} u_{\tau} / v$ corresponding to the mean dissipation rate presented are provided in Fig. 2(b). As expected, $\eta_{K}^{+}$increases with distance from the wall, and, again, the difference between the two flow regimes occurs when $y^{+} \gtrsim 350$.

The Taylor microscale, providing the intermediate length scale between the large- and small-scale statistics, was determined from

$$
\lambda \approx\left(\frac{30 v\left\langle u_{1}^{2}\right\rangle}{\langle\varepsilon\rangle}\right)^{0.5}
$$

Figure 2(c) shows the profiles of wall-normal dependence of the inner-scaled Taylor microscale, $\lambda^{+}=\lambda u_{\tau} / v$, for the two free-stream conditions. Unlike the Kolmogorov scale, the Taylor microscale changes very little across the boundary layer, and no difference is observed between the laminar and turbulent free-stream conditions. The corresponding Taylor-scale Reynolds number was estimated from

$$
\operatorname{Re}_{\lambda}=\frac{\lambda\left\langle u_{1}^{2}\right\rangle^{0.5}}{\sqrt{2} v}
$$

There is little variation in $R e_{\lambda}$, remaining between 100 and 120 , for $y^{+}<350$, with its maximum value occurring near the wall. Closer to the edge of the boundary layer, however, there is a rapid drop in $R e_{\lambda}$, slightly delayed for the turbulent free-stream for which $R e_{\lambda} \approx 40$.

A key scaling parameter for the large turbulent eddies is the scale $L$, and it is common practice to use the integral length scale to determine $L$. To find the integral length scale, we applied Taylor's hypothesis to the autocorrelation and integrated such that

$$
L=\frac{\left\langle U_{1}\right\rangle}{\left\langle u_{1}^{2}\right\rangle} \int_{0}^{\tau_{c}}\left\langle u_{1}(t+\tau) u_{1}(t)\right\rangle d \tau
$$

To minimize the impact of experimental bias and precision errors, which can result in slow convergence in the integral, the integration was conducted up to $\tau_{c}$, which was either the first zero-crossing of the autocorrelation or the first inflection point, whichever value was lower. The inner-scaled profiles of integral length scale $L^{+}=L u_{\tau} / v$ are shown in Fig. 2(e). In both laminar and turbulent free-streams, the integral length scale remains largely constant at $L^{+} \approx 0.4 \delta^{+}$. Note that for the turbulent free-stream case, $L^{+} \approx M^{+}$, and we should not expect to see much difference in the size of the large scales between the turbulent boundary layer and freestream turbulence. Note also that for the measurement points approaching and in the laminar free-stream, the value of $L$ was beyond the scale of Fig. 2(e) and is not shown, as the integral scale calculated only reflects long-wavelength oscillation in the free-stream conditions, as opposed to turbulent eddies. 
To find the corresponding large-scale Reynolds number, $R e_{L}=\left\langle\left|\delta_{L} u\right|\right\rangle L / v$, the average velocity increment was estimated through time averaging $\left|\delta_{L} u\right| \approx\left|u_{1}\left(t+L /\left\langle U_{1}\right\rangle\right)-u_{1}(t)\right|$ for all $t$. The resulting values of $R e_{L}$ are presented in Fig. 2(f) and are found to be $\mathcal{O}\left(R e_{\tau}\right)$ near the wall, decreasing toward the edge of the boundary layer. Interestingly, for most of the boundary layers, the velocity and length scales describing the large scales of turbulence, $\left|\delta u_{L}\right|$ and $L$, appear to be less affected by the free-stream turbulence conditions than the Kolmogorov scales.

In summary, the boundary layers with and without freestream turbulence demonstrate identical wall-normal dependence of large- and small-scale statistics near the wall, diverging toward the edge of the boundary layer where external intermittency becomes increasingly important.

As noted by Bailey and Witte, ${ }^{4}$ the integral length scale, $L$, is a poor indicator of the low-wavenumber boundary of the inertial cascade, as the calculation of $L$ is biased by the presence of non-local, potentially "inactive," 28 long-wavelength motions, for example, the very-large-scale and large-scale motions, e.g., Kim and Adrian, ${ }^{13}$ Guala et al.,${ }^{11}$ and Balakumar and Adrian. ${ }^{6}$ As a result, the scaling parameter $\eta_{0}$ is biased as well and becomes ineffective near the wall. Therefore we seek alternative descriptions for the large scales, which may be unbiased by the presence of these long-wavelength motions. In this respect, we note that it is possible to use dimensional arguments to define an alternative description of the large scales ${ }^{19}$ using turbulent kinetic energy $K$ and $\langle\varepsilon\rangle$ such that

$$
\mathcal{L}=\frac{K^{3 / 2}}{\langle\varepsilon\rangle} .
$$

Note that it is possible to modify this quantity to account for inhomogeneities through the introduction of an additional coefficient; ${ }^{17}$ however, that is not done here as these coefficients typically bring $\mathcal{L}$ closer to $L$, whereas we require a quantity that will describe the more isotropic large scales. In the present experiments, we use the isotropic approximation $K \approx 3 / 2\left\langle u_{1}^{2}\right\rangle$ to calculate $\mathcal{L}$ in order to investigate the possibility of using it as a surrogate to $L$ for describing the largest scales at the start of the energy cascade. A bias is likely to be introduced in our $K$ estimate by the anisotropy in the large scales, which will bias high in the turbulent boundary layer due to the streamwise normal Reynolds stress being higher than the other two normal components of the Reynolds stress. Hence $\mathcal{L}$ is likely to be slightly longer than would be found if the full three components of velocity were measured.

We also note that the estimate of $\mathcal{L}$ presented here assumes that the small scales are isotropic through the approach used for the calculation of $\langle\varepsilon\rangle$, necessitated by the measurements' inability to resolve the instantaneous velocity gradient tensor. However, the small scales may not be isotropic, as observed by Agostini and Leschziner, ${ }^{1}$ and also it is not expected that the small degree of anisotropy observed at small scales will have an appreciable impact on the calculation of $\mathcal{L}$.

Due to its dependence on $K$, which is a Reynolds number dependent quantity, there is some Reynolds number dependence in $\mathcal{L}$ which could impact the scaling of the dissipation scales. However, as can be observed in the study of Nedic et al., ${ }^{17}$ most of this Reynolds number dependence is confined to $R e_{\theta}<2000$; above this value of $R e_{\theta}$, there is very little Reynolds number dependence due to $\langle\varepsilon\rangle$ increasing proportionately with $K^{3 / 2}$.

In analogy to $R e_{L}$, we introduce

$$
\operatorname{Re}_{\mathcal{L}}=\frac{\left\langle\left|\delta_{\mathcal{L}} u\right|\right\rangle \mathcal{L}}{v},
$$

where $\delta_{\mathcal{L}} u$ is the longitudinal velocity increment, defined in Eq. (3), with $|r|=\mathcal{L}$. The wall-normal distribution of the innerscaled $\mathcal{L}$ and $R e_{\mathcal{L}}$ for the two flow regimes is presented in Figs. 2(g) and 2(h), respectively. This scale is slightly larger than the integral length scales, being closer to $\delta^{+}$, and displays more wall-normal dependence. Due to its dependence on $\left\langle u_{1}^{2}\right\rangle$, it drops significantly in the outer layer. In addition, there is effectively no dependence on free-stream conditions.

\section{SCALING OF LOCAL DISSIPATIVE SCALES}

We now seek to examine the scaling of the dissipative eddies within a turbulent boundary layer. As noted earlier, the scaling parameter, $\eta_{0}$, introduced by Yakhot and Sreenivasan ${ }^{31}$ scales with the local large-scale Reynolds number through $\eta_{0} \approx L R e_{L}^{-0.73}$ and is analogous to $\eta_{K} \sim L R e_{L}^{-0.75}$. Hence, how the local large scales, $L$, are determined can strongly influence the value of the scaling parameter $\eta_{0}$.

Bailey and Witte ${ }^{4}$ observed that $\eta_{0}$ failed to scale the PDFs of $\eta$ near the wall and instead introduced a lengthscale $L^{*}$ to characterize the largest nearly isotropic energyproducing eddies in a channel flow. They assumed a validity of Townsend's attached eddy hypothesis, ${ }^{28}$ which states that in wall-bounded flows, the scale of Reynolds-stress-contributing eddies depends on the distance from the wall, $y$, and cannot be larger than $y$ since these eddies are confined by the wall. They therefore suggested that $L^{*}=0.8 y$ as an appropriate length scale to describe the local, active contributions to the Reynolds stress and upper bound of the inertial subrange. There is no theoretical foundation for choosing the constant of proportionality 0.8 ; however, it was determined to be the most effective value when normalizing the dissipative motions for $y \lesssim 0.5 \delta$, the region where Townsend's attached eddy hypothesis has validity. Correspondingly, they defined

$$
R e_{L}^{*}=\frac{\left\langle\left|\delta_{L} u^{*}\right|\right\rangle L^{*}}{v},
$$

and anticipated that $L^{*}$ was a better descriptor for the energetic eddies at the upper limits of the universal equilibrium range and thus leads to a value of the local large-scale Reynolds number representing local contributions to Reynolds stress, particularly in the near-wall region of the boundary layer. Thus, $\eta^{*}=L^{*} R e_{L}^{*-0.73}$ would be a more appropriate scaling parameter for the small scales, which was found to be the case for $y / \delta<0.5$ in a channel flow. However, due to this limited range of applicability, we seek a better descriptor for the local large scales. Being based on isotropic approximations, $\mathcal{L}$ is potentially a better estimate for the top of the inertial subrange cascade than $L$ and should work everywhere in the boundary layer. Therefore, in this section, we investigate the scaling of the distribution of the dissipative scale, $\eta$, when using scaling 
parameters found by assuming that either $L, L^{*}$, or $\mathcal{L}$ are the appropriate descriptors for the large scales.

To find the distribution of $\eta$, we use Eq. (4) to define $\eta$ and find the PDF of these scales $Q(\eta)$. This PDF is found by evaluating the local Reynolds number $\left|\delta_{r} u\right| r_{1} / v$ throughout the measured time series and identifying instances where it is near unity. These instances are counted as an occurrence of a dissipation scale with $\eta=r_{1}$. Specifically, the $Q(\eta)$ distribution was calculated from each velocity time series using the following procedure, which was introduced in the study of Bailey et al. ${ }^{5}$ To do so, the values of $\left|u_{1}(t+\Delta t)-u_{1}(t)\right| U_{1} \Delta t / v$ were calculated for all $t$, resulting in a different value for each point in the time series. Then, the instances where this quantity was between 0.5 and 2 were counted as occurrences of dissipation at a scale $\eta=U_{1} \Delta t$. $\Delta t$ was then incremented by $1 / f_{s}$, and the process was repeated. These counts were obtained up to $U_{1} \Delta t$ $=4 L$ resulting in a count, $q(\eta)$, of the total number of occurrences of $\eta$ in the range $0<\eta<4 L$. Finally, the PDF $Q(\eta)$ was determined by normalizing such that

$$
Q(\eta)=\int_{0}^{4 L} q(\eta) d \eta=1 .
$$

This process was repeated for all $y$ positions within the boundary layer.

To investigate the suitability of the different descriptors of the large scales, three scaling parameters were used to normalize the PDFs of $\eta$ : (1) $\eta_{0}=L R e_{L}^{-0.73}$ as introduced by Yakhot and Sreenivasan ${ }^{31}$ and used by Schumacher ${ }^{24}$ and Hamlington et al. $;^{12}(2) \eta^{*}=L^{*} R e_{L}^{*-0.73}$ as suggested by Bailey and Witte; ${ }^{4}$ and (3) $\eta_{\mathcal{L}}=\mathcal{L} R e_{\mathcal{L}}^{*-0.73}$. The PDFs of $\eta$ determined from all $y$ measurement positions normalized by $\eta_{0}, \eta^{*}$, and $\eta_{\mathcal{L}}$ are presented in Figs. 3(a)-3(c), respectively, for the case without free-stream turbulence.
As expected, the general shape of the distributions of the PDFs is in good agreement with the previously reported distributions calculated both experimentally and numerically. Most notably, this is in the form of a skewed PDF biased toward the small scales, with a long tail toward the larger scales. For the most part, the maximum values of the PDFs are near $2.5 \eta_{0}$, $3 \eta^{*}$, and $2.2 \eta_{\mathcal{L}}$, respectively. However, it can be observed that each of the scalings displays regions of poor collapse, with the greatest deviations observed when the PDFs are scaled by $\eta_{0}$.

To provide a more detailed view of the degree of collapse near the wall under the different scalings, the PDFs for $y / \delta$ $<0.4$ are presented on linear axes in Figs. 4(a)-4(c). Consistent with the observations of Bailey and Witte, scaling by $\eta^{*}$ improves the collapse of the PDFs near the wall relative to that provided by $\eta_{0}$, indicating that the non-universality of the small scales and dependence on the large-scale shear observed by Morshed et al. ${ }^{16}$ and Hamlington et al. ${ }^{12}$ are due to the imperfect description of the large scales by $L$. When normalized by $\eta_{\mathcal{L}}$, there is a marked improvement relative to the $\eta_{0}$ scaled PDFs; however, it does not quite provide the same degree of collapse provided by $\eta^{*}$. This is most noticeable in the shift of the peak of $\eta / \eta_{\mathcal{L}}$.

To examine the dependency of the different scalings of the PDFs on the distance from the wall, the PDFs measured throughout the entire boundary layer are presented in the form of isocontours of probability in Figs. 4(d)-4(f) for the PDFs scaled by $\eta_{0}, \eta^{*}$, and $\eta_{\mathcal{L}}$. It can be observed from these isocontours that the greatest deviations from universal scaling do not appear near the wall, as occurs in a channel flow, but actually occur for $y^{+}>350$ (or, alternatively, $y / \delta>0.35$ ). In this range, the PDFs normalized by all three scaling parameters vary nonmonotonically, with the highest probabilities shifting to larger values than those observed near the wall as $y$ increases before
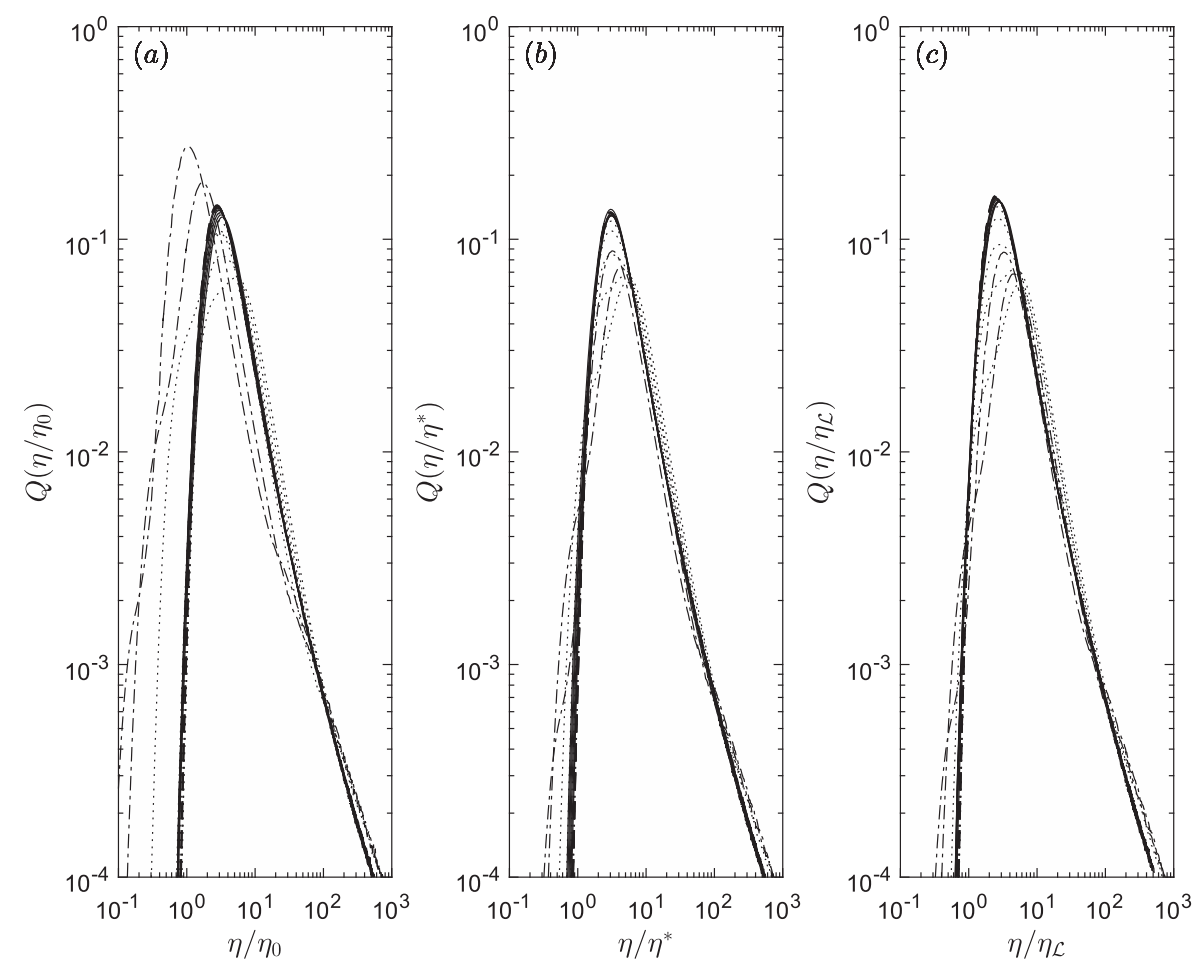

FIG. 3. PDFs of local dissipation scales from all measured positions within the boundary layer for the case with a laminar free stream, normalized by (a) $\eta_{0}$; (b) $\eta^{*}$; and (c) $\eta_{\mathcal{L}}$. 

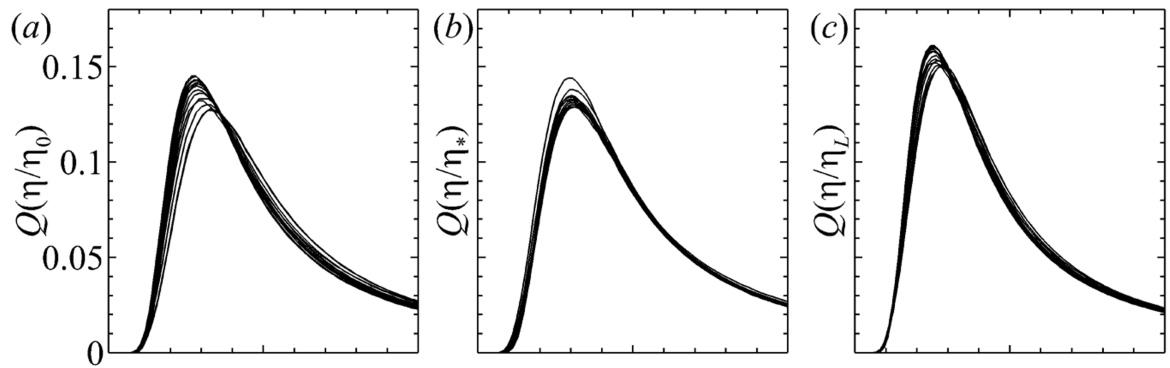

(d)
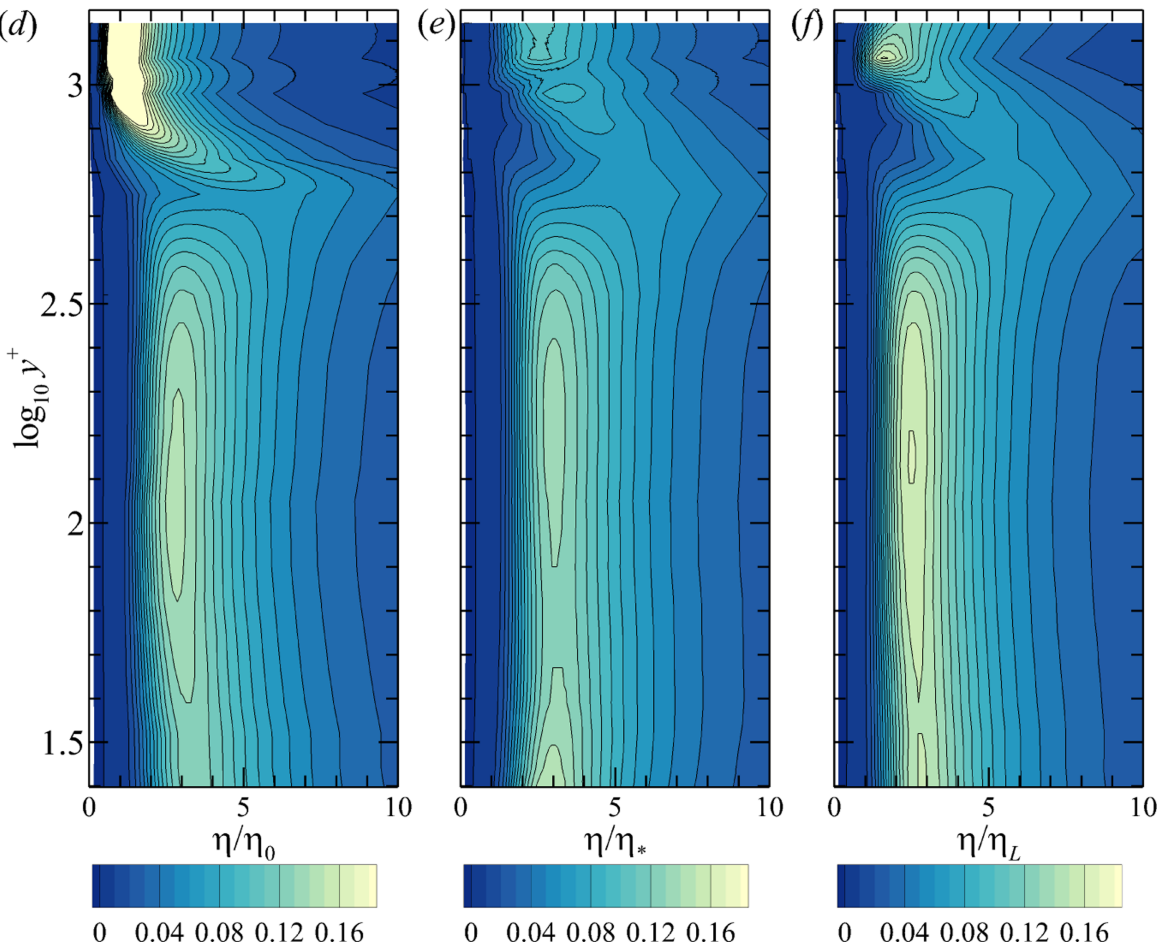

FIG. 4. Measured PDFs of local dissipation scales for the case without freestream turbulence using linear axes, normalized by (a) $\eta_{0}$; (b) $\eta^{*}$; and (c) $\eta_{\mathcal{L}}$ for $y / \delta<0.4$. The wall-normal dependence of the PDFs is shown normalized by (d) $\eta_{0}$; (e) $\eta^{*}$; and (f) $\eta_{\mathcal{L}}$. shifting to smaller values near the edge of the boundary layer. We attribute this non-universality to the effect of the external intermittency that exists in the wake region of the boundary layer. In this region, the flow will be intermittently laminar and turbulent, with the relative fraction of laminar to turbulent flow increasing toward the edge of the boundary layer. Hence, the PDFs of $\eta$ will be increasingly impacted as the instances of laminar flow in the time series increase in frequency and length toward the edge of the boundary layer and increasingly bias the calculation of $Q(\eta)$, which does not discriminate between laminar and turbulent flows.

To support such an intermittency argument, we can look at equivalent scaling of the PDFs for the case with free-stream turbulence. Although intermittent behavior is still present in this case, even when boundary layer turbulence is not present, there is still turbulence present in the free-stream fluid entrained into the boundary layer. Hence, the impact of the external intermittency on the PDFs of $\eta$ should be reduced. The PDFs measured for all $y$ positions when free-stream turbulence is present are shown in Figs. 5(a)-5(c) scaled by $\eta_{0}, \eta^{*}$, and $\eta_{\mathcal{L}}$, respectively. In all cases, there is improved agreement between the PDFs relative to that observed in Fig. 3, with the best agreement throughout the boundary layer and into the free stream being offered by the $\eta_{\mathcal{L}}$ scaling.
There is still some variation among the PDFs shown in Fig. 5, and we present the wall-normal dependence of this variation in Fig. 6, which shows the PDFs measured for $y / \delta<0.4$ in Figs. 6(a)-6(c) on linear axes and the wall-normal dependence throughout the boundary layer via the corresponding isocontours of the PDF value in Figs. 6(d)-6(f).

Figures 6(d)-6(f) display a much reduced degree of wallnormal dependence in the wake region for this case, consistent with the hypothesis that the lack of collapse in the PDFs observed in Fig. 3 was due to the presence of periods of laminar flow biasing the calculation of $\eta$ from the time series.

The results shown in Figs. 6(b) and 6(e) scaled by $\eta^{*}$ demonstrate the same improved collapse near the wall as in Figs. 4(b) and 4(e) when compared to the same PDFs scaled using $\eta_{0}$, consistent with the results of Bailey and Witte ${ }^{4}$ that indicate $\eta^{*}$ is a suitable scaling parameter when $y / \delta \lesssim 0.5$. However, also consistent with the results of Bailey and Witte, ${ }^{4}$ $\eta^{*}$ is increasingly unsuitable as a normalization parameter in the far-wall region $(y / \delta \gtrsim 0.5)$.

Conversely, although the $\eta_{\mathcal{L}}$ scaling does not work quite as well in the near-wall region as $\eta^{*}$, it does display improved collapse throughout the boundary layer, as shown in Figs. 5 and 6(f), with the far-wall scaling comparable to that provided by $\eta_{0}$. Near the wall, there is improvement relative to 

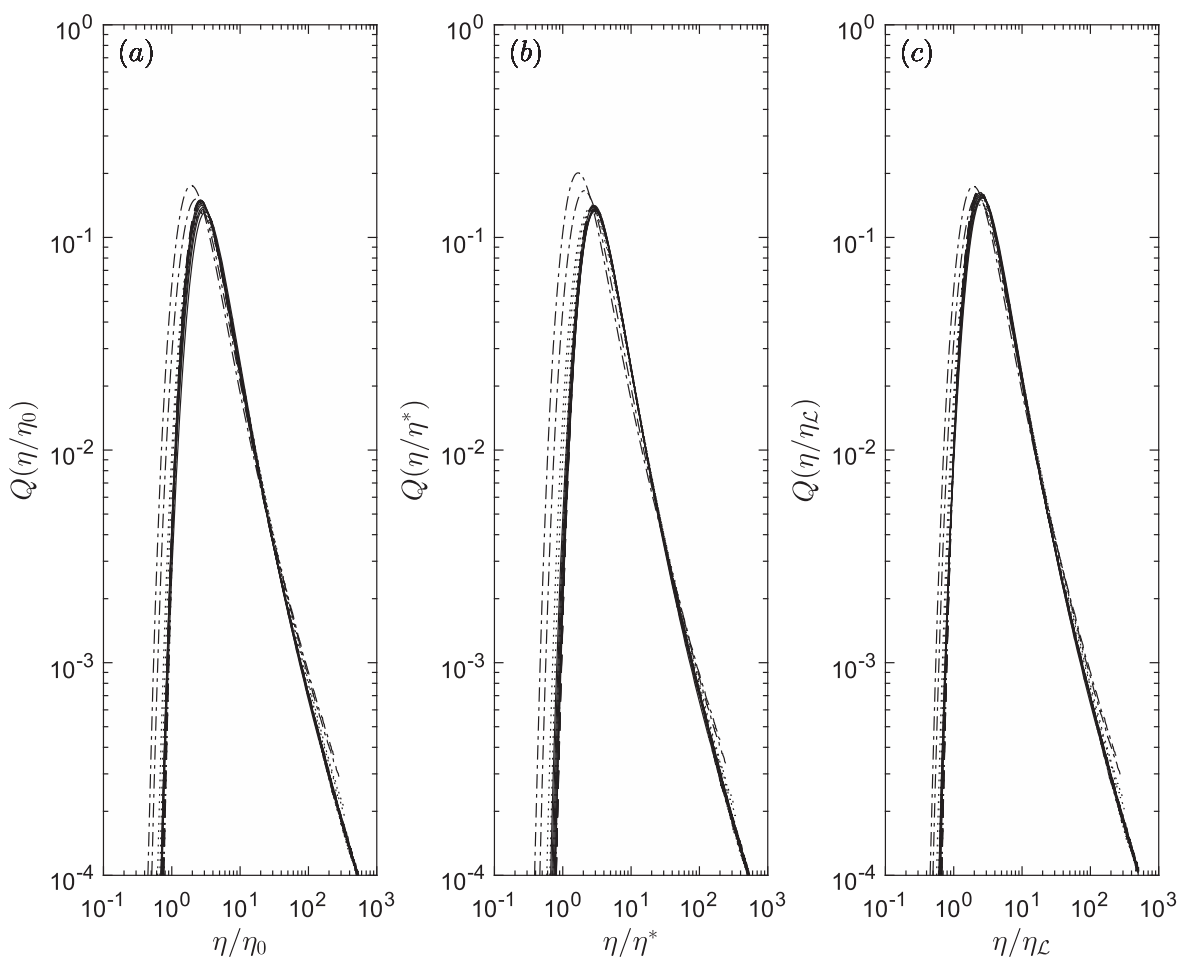

FIG. 5. PDFs of local dissipation scales from all measured positions within the boundary layer for the case with freestream turbulence, normalized by (a) $\eta_{0}$; (b) $\eta^{*}$; and (c) $\eta_{\mathcal{L}}$.
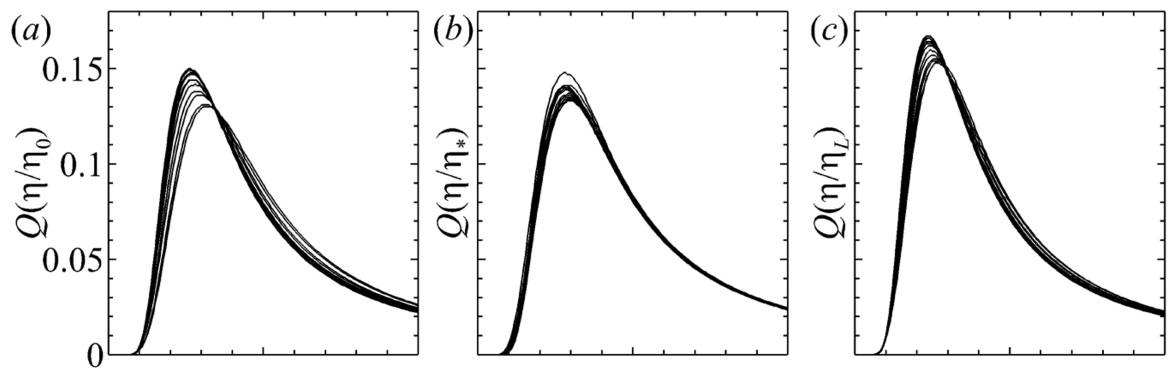

(d)

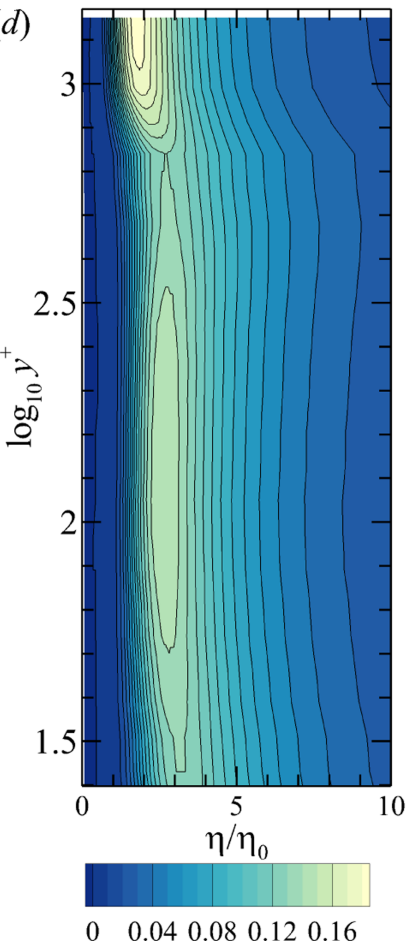

(e)

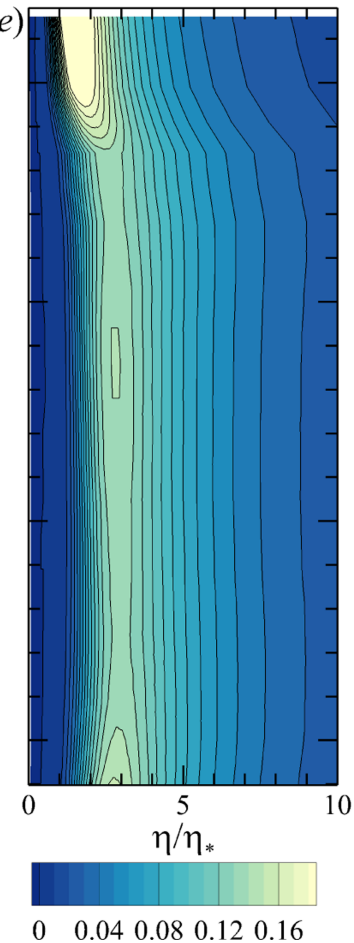

$(f)$

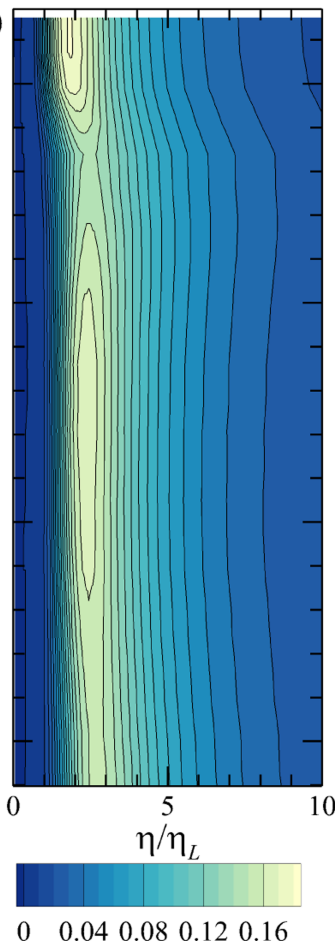

FIG. 6. Measured PDFs of local dissipation scales for the case with freestream turbulence using linear axes, normalized by (a) $\eta_{0}$; (b) $\eta^{*}$; and (c) $\eta_{\mathcal{L}}$ for $y / \delta<0.4$. The wall-normal dependence of the PDFs is shown, normalized by (d) $\eta_{0}$; (e) $\eta^{*}$; and (f) $\eta_{\mathcal{L}}$. 
$\eta_{0}$ when the PDFs are scaled by $\eta_{\mathcal{L}}$, with the near-wall scaling comparable to $\eta^{*}$.

\section{CONCLUSIONS}

Measurements of the turbulent boundary layer with and without free-stream turbulence were conducted at $R e_{\tau} \approx 1000$ using a thermal anemometry probe. The data were utilized to investigate the scaling behavior of the distribution of dissipative scales within the boundary layer turbulence. Specifically, the collapse of the probability density functions of the dissipative scales was examined using normalizing parameters built from three selected measures of the large scale turbulence. These were the measured integral length scale, an approximation based on Townsend's attached eddy hypothesis introduced by Bailey and Witte, ${ }^{4}$ and the length scale built from dimensional analysis of turbulent kinetic energy and dissipation rate.

The measured PDFs of $\eta$ were consistent with those observed in other flows. Although, unlike turbulent channel flows, in the outer region of the boundary layer, there was significantly reduced collapse in the scaled PDFs, irregardless of the scaling used. This lack of collapse was attributed to bias in the calculation of $\eta$ introduced by the intermittent presence of laminar flow in the time series. This attribution was supported by the significant improvement in the scaling of the probability density functions when the free-stream conditions were turbulent.

Within the near-wall region, the local large scale defined based on the distance from the wall was found to collapse the probability density functions, for the lower half of the boundary layer. This observation is consistent with prior observations of scaling within turbulent channel flows and supports the existence of a universal description for the small scales within external wall-bounded flows. However, this scaling does not extend to the outer region of the boundary layer, even for the case of a turbulent free stream. Instead it was found that scaling the PDFs using a parameter built from the turbulent kinetic energy and mean dissipation rate provided the best agreement throughout the entire depth of the boundary layer. Although it does not provide the same degree of collapse of the PDFs as the wall-dependent scaling, and relies on a priori knowledge of $\langle\epsilon\rangle$, since this quantity converges on the integral length scale for homogeneous isotropic turbulence, it should prove to be a more practical parameter to use in complex flows where the boundary layer thickness is not known.

\section{ACKNOWLEDGMENTS}

This research was partially supported by the Higher Committee for Education Development (HCED) in Iraq and the University of Misan in Misan, Iraq.

\footnotetext{
${ }^{1}$ Agostini, L. and Leschziner, M., "Spectral analysis of near-wall turbulence in channel flow at $\operatorname{Re}_{\tau}=4200$ with emphasis on the attached-eddy hypothesis," Phys. Rev. Fluids 2, 014603 (2017).

${ }^{2}$ Anselmet, F., Gagne, Y., Hopfinger, E., and Antonia, R., "High-order velocity structure functions in turbulent shear flow," J. Fluid Mech. 140, 63-89 (1984).
}

${ }^{3}$ Antonia, R., Chambers, A., and Phan-Thien, N., "Taylor's hypothesis and spectra of velocity and temperature derivatives in a turbulent shear flow," Boundary-Layer Meteorol. 19(1), 19-29 (1980).

${ }^{4}$ Bailey, S. and Witte, B., "On the universality of local dissipation scales in turbulent channel flow," J. Fluid Mech. 786, 234-252 (2015).

${ }^{5}$ Bailey, S. C. C., Hultmark, M., Schumacher, J., Yakhot, V., and Smits, A. J., "Measurements of the dissipation scales in turbulent pipe flow," Phys. Rev. Lett. 103, 014502 (2009).

${ }^{6}$ Balakumar, B. J. and Adrian, R. J., "Large- and very-large-scale motions in channel and boundary-layer flows," Philos. Trans. R. Soc., A 365, 665-681 (2007).

${ }^{7}$ Batchelor, G. and Townsend, A. A., "The nature of turbulent motion at large wave-numbers," Proc. R. Soc. A 199, 238-255 (1949).

${ }^{8}$ Champagne, F. H., "Fine-scale structure of turbulent velocity-field," J. Fluid Mech. 86, 67-108 (1978).

${ }^{9}$ Frisch, U., Turbulence: The Legacy of A. N. Kolmogorov (Cambridge University Press, 1995).

${ }^{10}$ Grant, H. L., Stewart, R. W., and Moilliet, A., "Turbulence spectra from a tidal channel," J. Fluid Mech. 12, 241-268 (1962).

${ }^{11}$ Guala, M., Hommema, S. E., and Adrian, R. J., "Large-scale and verylarge-scale motions in turbulent pipe flow," J. Fluid Mech. 554, 521-542 (2006).

${ }^{12}$ Hamlington, P., Krasnov, D., Boeck, T., and Schumacher, J., "Local dissipation scales and energy dissipation-rate moments in channel flow," J. Fluid Mech. 701, 419-429 (2012).

${ }^{13} \mathrm{Kim}$, K. C. and Adrian, R. J., "Very large-scale motion in the outer layer," Phys. Fluids 11(2), 417-422 (1999).

${ }^{14}$ Kolmogorov, A. N., "The local structure of turbulence in incompressible viscous fluid for very large Reynolds numbers," Dokl. Akad. Nauk SSSR 30, 301-305 (1941).

${ }^{15}$ Lumley, J., "Interpretation of time spectra measured in high-intensity shear flows," Phys. Fluids 8(6), 1056-1062 (1965).

${ }^{16}$ Morshed, N., Venayagamoorthy, S., and Dasi, L., "Intermittency and local dissipation scales under strong mean shear," Phys. Fluids 25, 011701 (2013)

${ }^{17}$ Nedić, J., Tavoularis, S., and Marusic, I., "Dissipation scaling in constantpressure turbulent boundary layers," Phys. Rev. Fluids 2(3), 032601 (2017).

${ }^{18}$ Paladin, G. and Vulpiani, A., "Degrees of freedom of turbulence," Phys. Rev. A 35, 1971-1973 (1987).

${ }^{19}$ Pope, S. B., Turbulent Flows (Cambridge University Press, 2000).

${ }^{20}$ Prasad, R. and Sreenivasan, K., "Quantitative three-dimensional imaging and the structure of passive scalar fields in fully turbulent flows," J. Fluid Mech. 216, 1-34 (1990).

${ }^{21}$ Prasad, R., Meneveau, C., and Sreenivasan, K., "Multifractal nature of the dissipation field of passive scalars in fully turbulent flows," Phys. Rev. Lett. 61(1), 74 (1988).

${ }^{22}$ Saddoughi, S. G. and Veeravalli, S. V., "Local isotropy in turbulent boundary layers at high Reynolds number," J. Fluid Mech. 268, 333-372 (1994)

${ }^{23}$ Schlatter, P. and Örlü, R., "Assessment of direct numerical simulation data of turbulent boundary layers,” J. Fluid Mech. 659, 116-126 (2010).

${ }^{24}$ Schumacher, J., "Sub-Kolmogorov-scale fluctuations in fluid turbulence," Europhys. Lett. 80, 54001-1-54001-6 (2007).

${ }^{25}$ Siggia, E., "Invariants for the one-point vorticity and strain rate correlation functions," Phys. Fluids 24(11), 1934-1936 (1981).

${ }^{26}$ Sreenivasan, K. R. and Antonia, R. A., "The phenomenology of smal-scale turbulence," Annu. Rev. Fluid Mech. 29, 435-472 (1997).

${ }^{27}$ Taylor, G. I., "The spectrum of turbulence," Proc. R. Soc. A 164(919), 476-490 (1938).

${ }^{28}$ Townsend, A. A., The Structure of Turbulent Shear Flow (Cambridge University Press, Cambridge, UK, 1976).

${ }^{29}$ Townsend, A. A., The Structure of Turbulent Shear Flow (Cambridge University Press, 1980).

${ }^{30}$ Yakhot, V., "Probability densities in strong turbulence," Phys. D 215(2), 166-174 (2006).

${ }^{31}$ Yakhot, V. and Sreenivasan, K., "Towards a dynamical theory of multifractals in turbulence," Phys. A 343, 147-155 (2004).

${ }^{32}$ Zhou, Q. and Xia, K.-Q., "Universality of local dissipation scales in buoyancy-driven turbulence," Phys. Rev. Lett. 104(12), 124301 (2010). 


\title{
Universality of local dissipation scales in turbulent boundary layer flows with and without free-stream turbulence
}

\author{
Sabah F. H. Alhamdi, and Sean C. C. Bailey
}

Citation: Physics of Fluids 29, 115103 (2017); doi: 10.1063/1.4996200

View online: https://doi.org/10.1063/1.4996200

View Table of Contents: http://aip.scitation.org/toc/phf/29/11

Published by the American Institute of Physics

\section{Articles you may be interested in}

Turbulent kinetic energy budgets in wall bounded flows with pressure gradients and separation

Physics of Fluids 29, 115108 (2017); 10.1063/1.4992793

From two-dimensional to three-dimensional turbulence through two-dimensional three-component flows Physics of Fluids 29, 111101 (2017); 10.1063/1.4990082

Attenuation of turbulence by the passive control of sweep events in a turbulent boundary layer using microcavities

Physics of Fluids 29, 115102 (2017); 10.1063/1.4995466

Introduction to Focus Issue: Two-Dimensional Turbulence

Physics of Fluids 29, 110901 (2017); 10.1063/1.5012997

A two-dimensional toy model for geophysical turbulence

Physics of Fluids 29, 111114 (2017); 10.1063/1.4985990

Analysis of flapping motion of reattaching shear layer behind a two-dimensional backward-facing step Physics of Fluids 29, 115104 (2017); 10.1063/1.4996622

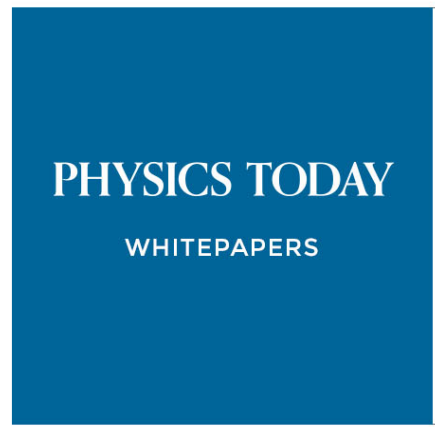

ADVANCED LIGHT CURE ADHESIVES

Take a closer look at what these environmentally friendly adhesive systems can do

\section{READ NOW}

PRESENTED BY

Q MASTERBOND: 